\title{
Rotation \& differential rotation of the active Kepler stars
}

\author{
Timo Reinhold ${ }^{1}$, Ansgar Reiners ${ }^{2}$ and Gibor Basri ${ }^{3}$ \\ ${ }^{1,2}$ Institut für Astrophysik, Universität Göttingen, Friedrich-Hund-Platz 1, \\ 37077 Göttingen, Germany \\ ${ }^{1}$ email: reinhold@astro.physik.uni-goettingen.de \\ 2 email: areiners@astro.physik.uni-goettingen.de \\ ${ }^{3}$ Astronomy Department, University of California, Berkeley, CA 94720, USA \\ email: basri@berkeley.edu
}

\begin{abstract}
Stellar rotation is a well-known quantity for tens of thousands of stars. In contrast, differential rotation (DR) is only known for a handful of stars because DR cannot be measured directly. We present rotation periods for more than 24,000 active stars in the Kepler field. Thereof, more than 18,000 stars show a second period, which we attribute to surface differential rotation. Our rotation periods are consistent with previous measurements and the theory of magnetic braking. Our results on DR paint a rather different picture: The temperature dependence of the absolute shear $\Delta \Omega$ is split into two groups separated around $6000 \mathrm{~K}$. For the cooler stars $\Delta \Omega$ only slightly increases with temperature, whereas stars hotter than $6000 \mathrm{~K}$ show large scatter. This is the first time that DR has been measured for such a large number of stars.
\end{abstract}

Keywords. Rotation, differential rotation, Kepler, activity

\section{Introduction}

Stellar rotation strongly correlates with the age of the star and its level of activity. Active stars use to have a strong magnetic field responsible for various activity phenomena, e.g., spots, flares, etc.. Differential rotation (DR) triggers the magnetic field generation of the Sun $(\alpha \Omega$-dynamo) and is believed to be a major ingredient of the field generation in other active stars. Stellar variability due to star spots can be used as tracer of the stellar rotation period, esp. offering the possibility to detect DR. The Kepler space telescope delivers high quality data, with unprecedented duty cycle and number of targets, making it an ideal source for the search of periodic stellar variability.

In this study we summarize the most important results from Reinhold et al. (2013). We present rotation period measurements for more than 24,000 stars in the Kepler field. More than $75 \%$ of them showed a second period with we attribute to surface differential rotation. Furthermore, we show that our rotation periods and DR measurements are consistent with previous rotation measurements and theoretical predictions from mean-field theory, respectively.

\section{Analysis}

We analyzed Kepler Q3 data, which was reduced by the PDC-MAP pipeline to search for periodic stellar variability. Active stars were selected from the whole sample by filtering out stars whose range of variability amplitude $R_{v a r}>0.3 \%$ (see Basri et al. 2010; Basri et al. 2011). To extract the most significant periods from a light curve we used 


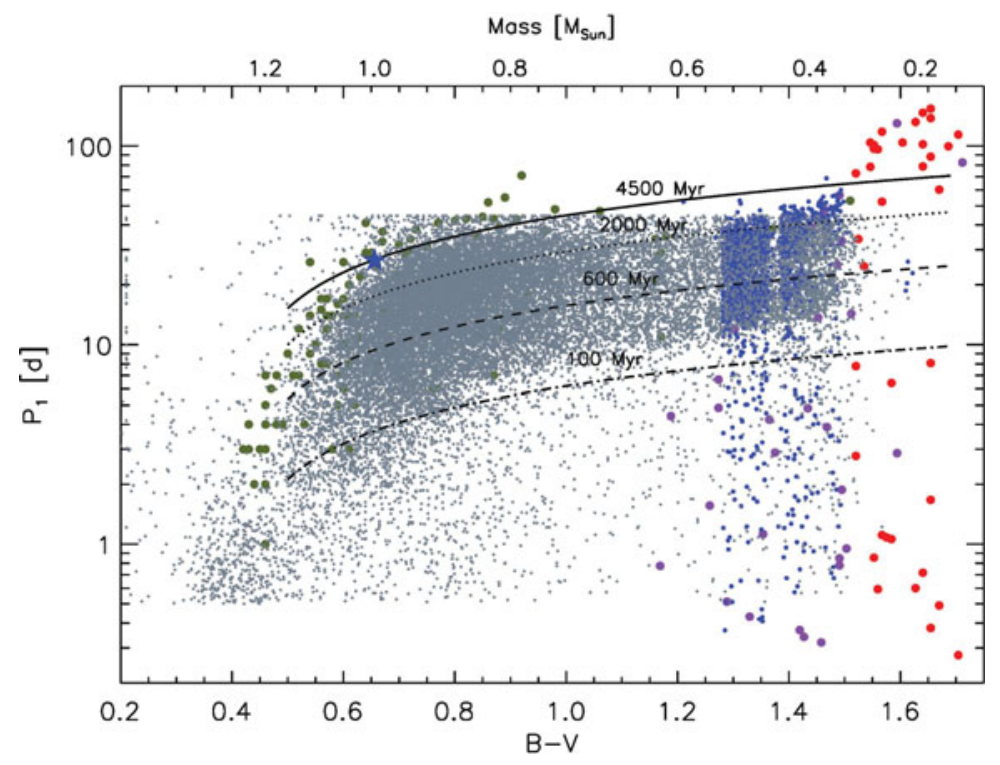

Figure 1. $B-V$ color vs. rotation period $P_{1}$ of the 24,124 stars with at least one detected period (gray dots). The filled circles represent data from Baliunas (1996) (olive), Kiraga, M. \& Stepien, K. (2007) (purple), Irwin et al. (2011) (red), and McQuillan et al. (2013) (blue). Towards cooler stars we found an increase in rotation periods with a steep rise around $B-V \approx 0.6$ supplying evidence of rotational braking. The black lines represent a color-period relation found by Barnes (2007) for different isochrones. The blue star marks the position of the Sun for comparison.

the Lomb-Scargle periodogram in a prewhitening approach, yielding five periods for a global sine fit. From the global sine fit we selected the period with the highest power found in the prewhitening process, and call it $P_{1}$. Furthermore, we applied different filters, for instance, a lower and upper limit according $0.5 d<P_{1}<45 d$. This procedure yielded 24,124 rotation periods $P_{1}$. If a second period within $\pm 30 \%$ of $P_{1}$ was found by our prewhitening analysis we called this period $P_{2}$. In 18,616 cases we found a second period $P_{2}$, which we attribute to surface DR. For details of the method and the selection process we refer the reader to Reinhold \& Reiners (2013) and Reinhold et al. (2013).

\section{Results}

Rotation periods of the 24,124 stars are shown in Fig. 1. We see that our results are consistent with previous measurements. A strong increase of the rotation periods is evident around $B-V \approx 0.6$ supplying evidence of rotational braking. Furthermore, our results show good agreement with isochrones supplied by Barnes (2007) using empirical relations between color (mass) and rotation period.

For all stars with two detected periods, $P_{1}$ and $P_{2}$, we calculated the absolute surface shear $\Delta \Omega:=2 \pi\left|1 / P_{1}-1 / P_{2}\right|$ as a measure of DR. We show the dependence of $\Delta \Omega$ on effective temperature in Fig. 2. In contrast to Barnes et al. (2005) we cannot reproduce the strong temperature dependence of $\Delta \Omega$ over the whole temperature range. For cool stars $(3500-6000 \mathrm{~K})$ we find that $\Delta \Omega$ shows weak dependence on temperature with a shallow increase towards hot stars. A different behavior of $\Delta \Omega$ is found for stars hotter than $6000 \mathrm{~K}$. Our measurements show large scatter with a strong increase of $\Delta \Omega$ with temperature. These trends can be seen best by looking at the mean values $\langle\Delta \Omega\rangle$ of our measurements for different temperature bins, shown as open black circles. 


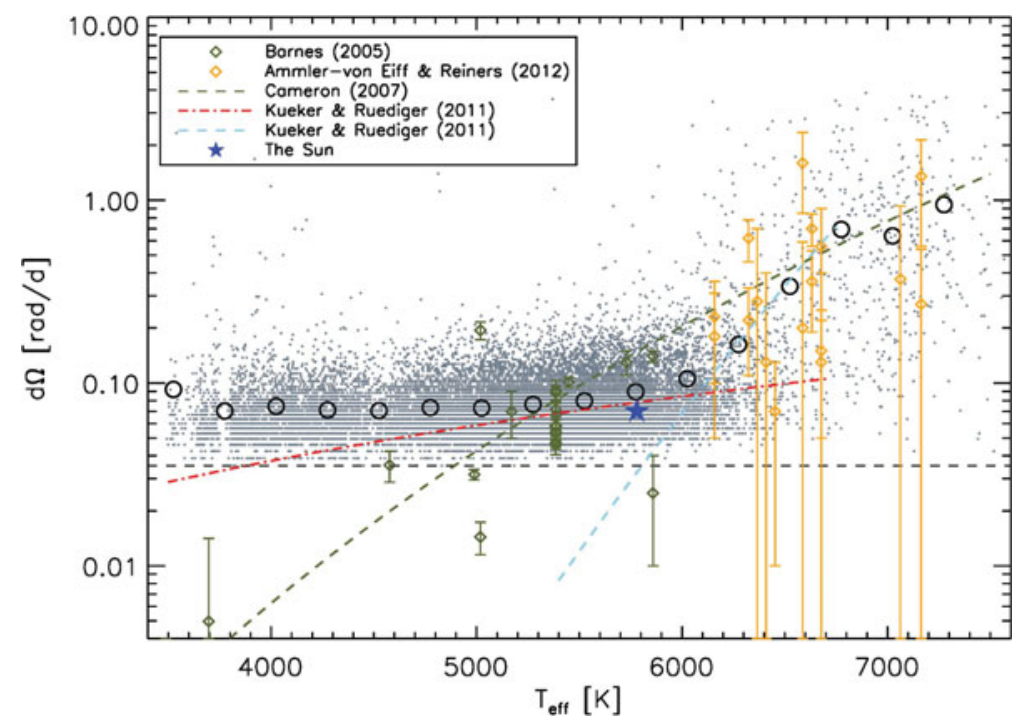

Figure 2. Effective temperature vs. horizontal shear $\Delta \Omega$ summarizing different measurements: The olive diamonds and error bars were taken from Barnes et al. (2005), the olive dashed curve from Collier Cameron (2007). Orange diamonds and error bars show measurements from Ammler-von Eiff \& Reiners (2012). Our measurements are shown as gray dots. The red dash-dotted line and the light blue dashed line show theoretical predictions from Küker \& Rüdiger (2011). The black dashed line marks our detection limit. The blue star marks the position of the Sun for comparison. The open black circles represent weighted means of our measurements for different temperature bins. From 3500-6000 K, $\Delta \Omega$ shows only weak dependence on temperature. Above $6000 \mathrm{~K}$ the shear strongly increases as reported by Barnes et al. (2005) and Collier Cameron (2007). The different behavior of $\Delta \Omega$ in these two temperature regions was supported by theoretical predictions from Küker \& Rüdiger (2011) (red and light blue lines).

Furthermore, we see that these trends are in good agreement with theoretical prediction from mean-field theory by Küker \& Rüdiger (2011). These authors found that the temperature dependence of $\Delta \Omega$ cannot be represented by a single power law over the whole temperature range but should rather be fitted separately for two groups separated around $6000 \mathrm{~K}$ (red dash-dotted and light blue dashed line).

For stars hotter than $7000 \mathrm{~K}$ the results should be treated with caution because there might probably be a contamination by long-period pulsators, e.g., $\gamma$ Dor or extreme $\delta$ Scuti stars among our periods. It is challenging to distinguish between pulsations and rotation periods shorter than half a day only from the light curve.

This task will partly be covered in our future work when we tackle the full Kepler time coverage to see how stable the derived periods are over many years. Analyzing the remaining quarters of Kepler data will definitely shrink our sample, but will also provide a condensed set of reliable rotation periods of tens of thousands of stars.

\section{References}

Ammler-von Eiff, M. \& Reiners, A. 2012, A\&A, 542, A116

Baliunas, S., Sokoloff, D., \& Soon, W. 1996, ApJ, 457, L99

Barnes, J. R., Collier Cameron, A., Donati, J.-F., James, D. J., Marsden, S. C., \& Petit, P. 2005, MNRAS, 357 ,L1

Barnes, S. A. 2007, ApJ, 669, 1167 
Basri, G., Walkowicz, L. M., Batalha, N., Gilliland, R. L., Jenkins, J., Borucki, W. J., Koch, D., Caldwell, D., Dupree, A. K., Latham, D. W., Meibom, S., Howell, S., \& Brown, T. 2010, ApJ, 713, L155

Basri, G., Walkowicz, L. M., Batalha, N., Gilliland, R. L., Jenkins, J., Borucki, W. J., Koch, D., Caldwell, D., Dupree, A. K., Latham, D. W., Marcy, G. W., Meibom, S., \& Brown, T. 2011, $A J, 141,20$

Collier Cameron, A. 2007, AN, 328, 1030

Irwin, J., Berta, Z. K., Burke, C. J., Charbonneau, D., Nutzman, P., West, A. A., \& Falco, E. E. 2011, ApJ, 727, 56

Kiraga, M. \& Stepien, K. 2007, Acta Astron., 57, 149

Küker, M. \& Rüdiger, G. 2011, AN, 332, 933

McQuillan, A., Aigrain, S., \& Mazeh, T. 2013, MNRAS, 432, 1203

Reinhold, T. \& Reiners, A. 2013, A\& A, 557, A11

Reinhold, T., Reiners, A., \& Basri, G. 2013, ArXiv e-prints 